\title{
Dynamic Response of Pavement Plates to the Positive and Negative Phases of the Friedlander Load
}

\author{
S. W. Alisjahbana, ${ }^{\text {a }}$ Safrilah, ${ }^{\mathrm{a}}$ J. C. P. Putra, ${ }^{\mathrm{a}}$ A. Asmi, ${ }^{\mathrm{a}}$ I. Alisjahbana, ${ }^{\mathrm{b}}$ S. Kiryu, ${ }^{\mathrm{c}}$ and \\ B. S. Gan ${ }^{\mathrm{d}}$
}

${ }^{a}$ Civil Engineering Department, Bakrie University, Jakarta, Indonesia

${ }^{\mathrm{b}}$ Civil Engineering Department, Stanford University, Stanford, USA

${ }^{c}$ Department of Architecture, Graduate School of Engineering, Nihon University, Koriyama, Japan

${ }^{\mathrm{d}}$ Department of Architecture, College of Engineering, Nihon University, Koriyama, Japan

The dynamic response of pavement plates to a localized Friedlander load based on the threeparameter foundation model with the account of soil inertia is analyzed. The pavement plate is represented by a thin orthotropic plate of finite dimensions, which can rotate and transfer deformation along the contour. The subgrade is simulated with the Pasternak foundation model, including the inertia soil factor, the localized dynamic load is simulated with the Friedlander decay function allowing for the positive and negative phases; with the time distribution described by the Dirac function. The governing equation of the problem is solved with the modified Bolotin method for determining the natural frequencies and mode numbers of the system. The Mathematica program is used to define the natural frequencies of the system from the transcendental equations. Analysis results for several parameters related to the dynamic response of plates to a localized dynamic load, which includes both positive and negative phases, are presented. The impact of the Friedlander load with the negative phase added on the response of the pavement plate is numerically simulated.

Keywords: pavement plate, Friedlander load, Pasternak foundation, positive and negative phases, modified Bolotin method.

Introduction. Dynamic response of a thin orthotropic plate subjected to a dynamic load is important, not only for pavement design but also for many other applications [1]. For instance, Uzan and Lytton [2] used measured pavement dynamic response information to investigate pavement non-destructive evaluation. Another application can be found in military countermine detection, where an unmanned automatic vehicle equipped with landmine detection sensors transverses minefield to identify locations of potential landmine [3]. A significant difference between static theory and its dynamic counterpart is that inertial effect, ignored in the former, is taken into account in the latter [4]. To better understand the dynamic response of a thin orthotropic plate subjected to a dynamic load, it is necessary to analyze the dynamic effects of a dynamic load on a concrete pavement. In 1954, Kenney [5] studied the steady-state response of a moving load on a beam on elastic foundation. Some studies using the finite element method to solve the response of a thin plate subjected to dynamic loads with applications in pavement design and non-destructive evaluation have been developed earlier [6-8]. More recently, finite element procedures have been developed to carry out the response of continuous pavements of finite dimensions to moving vehicle loads [6,9]. In the studies mentioned above, the rigid pavements are modeled as orthotropic plates sitting on Winkler soil type. However, in engineering practice, the effect of foundation inertia on the central deflection of the plate has significant effects on the dynamic response of the plate modeling the pavement [10]. For this reason, Gibigaye et al. [11] take into account the inertia of the foundation soil on the dynamic response of civil engineering structures. 
This work investigates the dynamic analytical response of pavement plate subjected to localized Friedlander dynamic loads sitting on an inertial soil. The rigid pavement is modeled as a thin plate, allowing the rotations and the vertical deformations along its edges. To take into account its inertia, the soil is modeled as a three-parameter type of soil [11]. The free vibration solution of the problem is solved by the method of separation of variables so that the superposition gives a solution satisfying the boundary conditions. The pavement plate deflection is expressed as eigenfunction products, that is obtained based on the orthogonality properties of eigenfunctions [12]. The localized Friedlander dynamic load is expressed as the Dirac delta function [13]. Particular emphasis is focused on the evaluation of midpoint displacements of the concrete pavement plates subjected to the Friedlander decaying function which includes both positive and negative phase of loading with different thickness and the varied position of the localized load.

1. Description of the Pavement Plates. Consider a rectangular pavement plate of thickness $h$ sitting on the three-parameter type foundation model $\left(k, G_{s}\right.$, and $\left.m_{0}\right)$ within the linear elastic region. The origin of the Cartesian coordinate system is located in the mid-plane with the $z$-axis perpendicular to the plane as shown in Fig. 1. The $a$ and $b$ are dimensions along the $x$ - and $y$-axes, respectively.

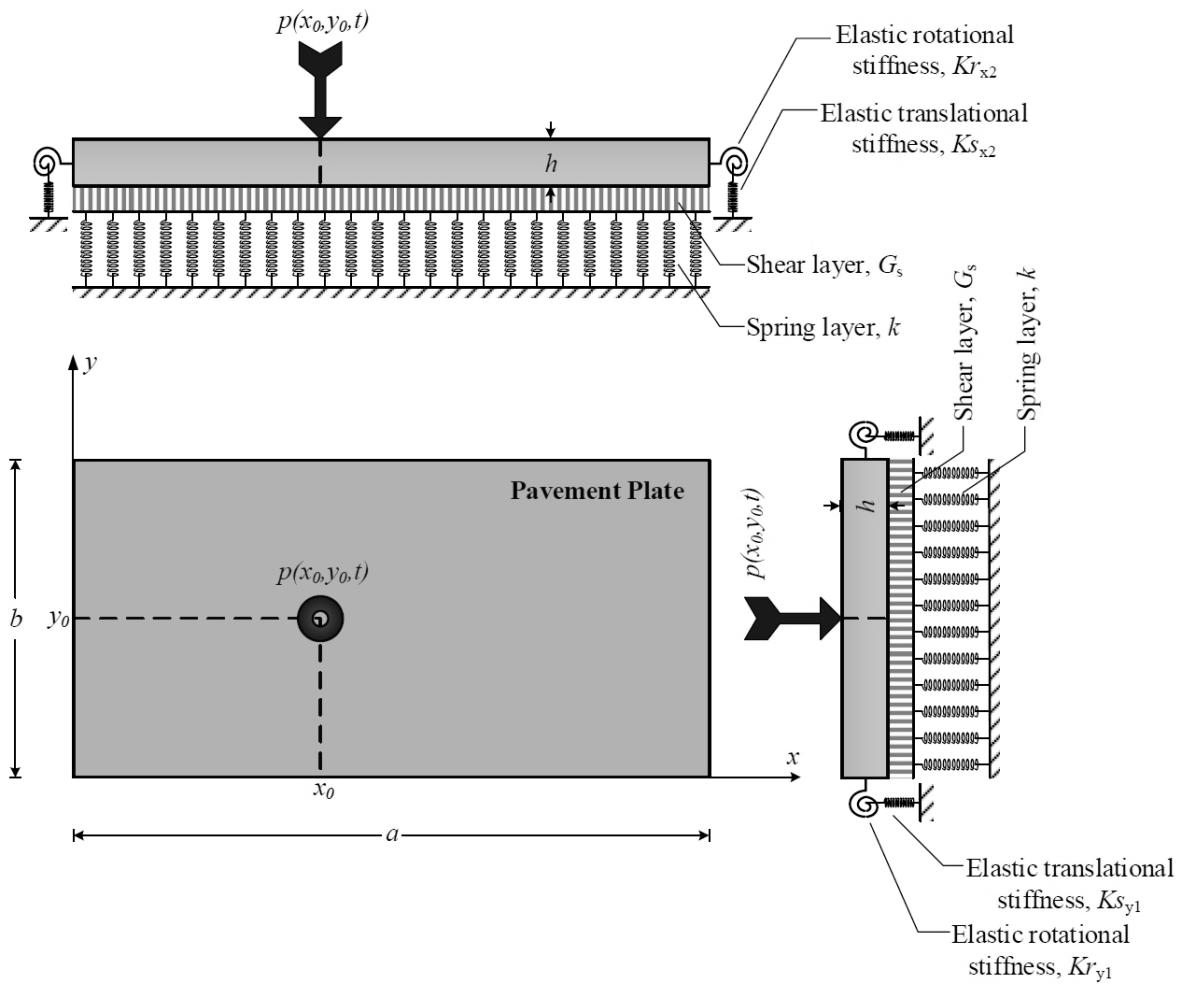

Fig. 1. The geometry of the pavement concrete plate supported by a three-parameter type foundation model.

According to the classic theory of thin plates and taking into account the reduced mass of soil, the deflection of the concrete pavement plate is governed by the following partial differential equation [11]:

$$
D_{x} \frac{\partial^{4} w(x, y, t)}{\partial t^{4}}+2 B \frac{\partial^{4} w(x, y, t)}{\partial x^{2} \partial y^{2}}+D_{y} \frac{\partial^{4} w(x, y, t)}{\partial y^{4}}+k w(x, y, t)-G_{s} \nabla^{2} w(x, y, t),
$$




$$
\left(\rho+m_{0}\right) h \frac{\partial^{2} w(x, y, t)}{\partial t^{2}}+\gamma h \frac{\partial w(x, y, t)}{\partial t}=p(x, y, t),
$$

where $w(x, y, t)$ is the vertical deflection of the orthotropic plate at point $(x, y)$ and time $t$, $D_{x}$ is the flexural rigidity of plate in the $x$-direction, $B$ is the torsional rigidity, $D_{y}$ is the flexural rigidity of the plate in the $y$-direction, $k$ is the stiffness coefficient of the elastic foundation, $G_{s}$ is the shear modulus of the shear layer of the elastic foundation, $\rho$ is the mass density of the plate, $m_{0}$ is a linear reduced mass of the foundation stiffness coefficient, $h$ is the thickness of the plate, and $\gamma$ is the critical damping ratio of the plate.

2. Localized Friedlander Loading. Figure 2 shows a localized Friedlander decaying loading function which includes the positive and the negative phases, $P(t)$, that can be expressed by the following equations $[14,15]$ :

$$
\begin{gathered}
P(t)=p_{r, \max }\left(1-\frac{t}{t_{d}}\right) e^{-\alpha \frac{t}{t_{d}}} \quad \text { for } \quad 0 \leq t \leq t_{d}, \\
P(t)=-p_{r, \min }\left(\frac{6.75\left(t-t_{d}\right)}{t_{d-}}\right)\left(1-\frac{\left(t-t_{d}\right)}{t_{d-}}\right)^{2} \text { for } t_{d} \leq t \leq t_{d}+t_{d-} .
\end{gathered}
$$

Here, $p_{r, \max }$ is the peak pressure, $t_{d}$ is positive phase duration, $\alpha$ is a waveform parameter, $p_{r, \min }$ is the negative peak pressure, and $t_{d-}$ is negative phase duration.

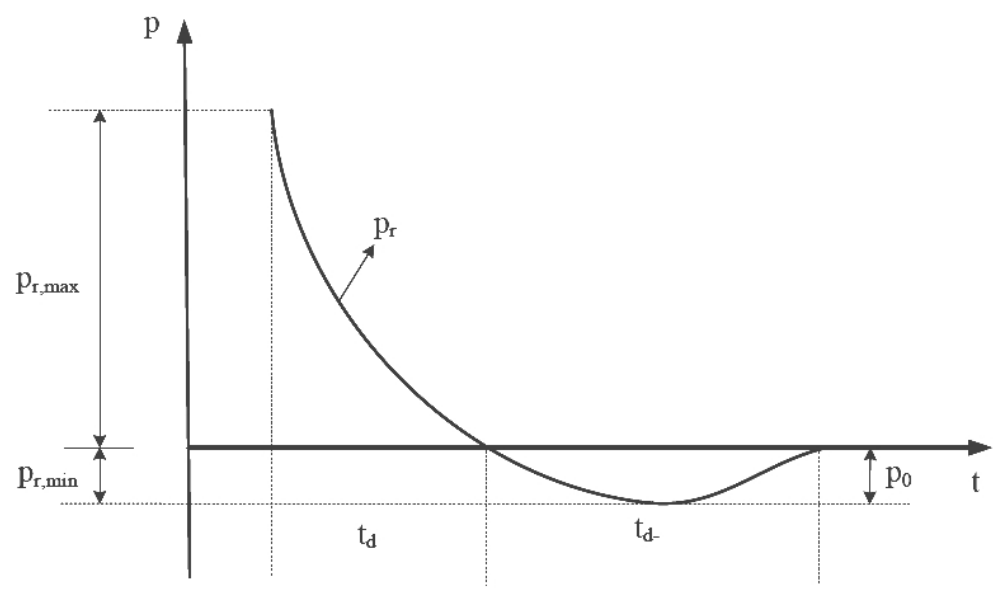

Fig. 2. The localized Friedlander decaying load.

The localized dynamic loading is a function of position and time, $p(x, y, t)$, that can be expressed by using the Dirac delta function, $\delta[\cdot]$, as follows:

$$
p(x, y, t)=P(t) \delta[x-x(t)] \delta[y-y(t)]=P(t) \delta\left[x-x_{0}\right] \delta\left[y-y_{0}\right],
$$

where $P(t)$ is the localized dynamic loading, which can be expressed by Eqs. (2) and (3), $x(t)$ is the position of the localized dynamic loading in the $x$-direction, $y(t)$ is the position of the localized dynamic loading in the $y$-direction, $x_{0}$ is the initial position of the dynamic loading in the $x$-direction, and $y_{0}$ is the initial position of the localized dynamic loading in the $y$-direction. 
3. Vibration Analysis. In the first part of this paper, the free vibration of pavement concrete plate on a Pasternak foundation, including the $m_{0}$ factor as a soil parameter, is studied using Levy's solution. The free vibration solution of the system is set as

$$
w(x, y, t)=W(x, y) \sin \omega t=X(x) Y(y) \sin \omega t,
$$

where $W(x, y)$ is only a function of the position coordinates and $\omega$ is the circular frequency of the system.

The eigenfrequencies of the systems can be solved from the undamped free vibration of Eq. (1) by postulating that the eigenfrequencies are analogous to the case of a plate with simple support at all edges, which can be expressed as

$$
\omega_{m n}^{2}=\frac{1}{\left(\rho h+m_{0}\right)}\left(D_{x} \frac{p^{4} \pi^{4}}{a^{4}}+2 B \frac{p^{2} q^{2} \pi^{4}}{a^{2} b^{2}}+D_{y} \frac{q^{4} \pi^{4}}{b^{4}}+k+G_{s}\left(\frac{p^{2} \pi^{2}}{a^{2}}+\frac{q^{2} \pi^{2}}{b^{2}}\right)\right),
$$

where $p$ and $q$ are real numbers to be solved from a system of two transcendental equations, obtained from the solution of two auxiliary Levy's type problems, also known as the modified Bolotin method [16].

3.1. First and Second Auxiliary Levy-Type Problems. The solution of the free vibration problem in Eq. (6) can be obtained from

$$
W(x, y)=X_{m n}(x) \sin \left(\frac{q \pi y}{b}\right),
$$

which satisfies the elastic vertical translation $\left(k s_{x 1}, k s_{x 2}\right)$ and rotational $\left(k r_{x 1}, k r_{x 2}\right)$ boundary conditions [12]. The solutions of the characteristic equation become

$$
X_{m n}(x)=A_{1} \cos \left(\frac{p \pi}{a} x\right)+A_{2} \sin \left(\frac{p \pi}{a} x\right)+A_{3} \cosh \left(\frac{\beta \pi}{a b} x\right)+A_{4} \sinh \left(\frac{\beta \pi}{a b} x\right),
$$

where

$$
\beta=\sqrt{\frac{2 B q^{2} a^{2}}{D_{x}}+p^{2} b^{2}+\frac{G_{s} a^{2} b^{2}}{\pi^{2} D_{x}}} .
$$

Equation (8) gives the general form of the eigenmode of the pavement plate in the $x$-direction. Substituting the eigenmode according to Eq. (8) into the boundary conditions along $x=0$ and $x=a$, results in the linear algebra equation in terms of $A_{i}$, as follows:

$$
[\mathbf{a}]\{\mathbf{A}\}^{T}=\{\mathbf{0}\}^{T},
$$

where $a_{i j}$ are the coefficients of [a].

To obtain a non-trivial solution, it is necessary to propose that the determinant of Eq. (9) is zero. The determinant of Eq. (9) is called the transcendental equation of the first auxiliary Levy's type problem. The second auxiliary Levy-type problem in the $y$-axis can be determined analogously to the above formulations.

3.2. Determination of Mode Numbers. The mode numbers $p$ and $q$, respectively, in the $x$ - and $y$-directions of the system can be solved by solving the transcendental equations simultaneously. The solutions cannot be determined analytically. Hence the Mathematica 
software is used to obtain solutions numerically. The eigenfunction of the orthotropic plate is therefore given by

$$
W(x, y)=\sum_{m=1}^{\infty} \sum_{n=1}^{\infty} X_{m n}(x) Y_{m n}(y) .
$$

3.3. Determination of the Time Function $T_{m n}(t)$. The general solution in the time domain is given by

$$
T_{m n}(t)=\frac{\int_{0}^{a} X_{m n}(x) d x \int_{0}^{b} Y_{m n}(y) d y}{\rho h Q_{m n} \omega_{m n}\left(\sqrt{1-\gamma^{2}}\right) t} \int_{0}^{t}\left(p(y, y, \tau) e^{-\gamma \omega_{m n}(t-\tau)} \sin \omega_{m n} \sqrt{1-\gamma^{2}}(t-\tau)\right) d \tau
$$

where $p(x, y, t)$ is the localized Friedlander decaying function dynamic loading, respectively, for positive phase according to Eq. (3) and for negative phase according to Eq. (4). Finally, the dynamic deflection of the pavement plate on Pasternak foundation models with the inclusive of the inertial soil factor is found by multiplying Eqs. (10) and (11).

4. Numerical Results and Discussion. Numerical calculations are carried out for different parameters of the problem. The number of modes in the $x$ - and $y$-directions is taken as $m=1,2, \ldots, 5$ and $n=1,2, \ldots, 5$ by considering the convergence of the eigenvalues. In this work, a finite rectangular pavement plate sitting on the three-type parameter foundation model is considered as shown in Fig. 1. The size of the pavement plate is $5 \times 3.5 \mathrm{~m}$. For comparison sake, two values of the plate thicknesses are considered: $h=0.18$ and $0.20 \mathrm{~m}$. The physical characteristics of the pavement plate are: density $\rho=2500 \mathrm{~kg} / \mathrm{m}^{3}$, Poisson's ratios $v_{x}=0.2$ and $v_{y}=0.3$, the elastic moduli $\left(E_{x}\right.$ and $\left.E_{y}\right)$ of the concrete plate is $27.8 \cdot 10^{6}$ and $30 \cdot 10^{6} \mathrm{~Pa}$, respectively. The boundary conditions properties of the pavement plate are: $k s_{x 1}=k s_{x 2}=k s_{y 1}=k s_{y 2}=250 \mathrm{MN} / \mathrm{m}$ and $k r_{x 1}=$ $=k r_{x 2}=k r_{y 1}=k r_{y 2}=1.0 \mathrm{~N} \cdot \mathrm{m} / \mathrm{rad}$ [17]. It is also assumed that the damping ratio of the system equals $\gamma=5 \%$. Two values of soil parameters are considered: Case $1, H_{s}=0.5 \mathrm{~m}$, $k=166.2 \mathrm{MN} / \mathrm{m}, G_{s}=2.596 \mathrm{MN} / \mathrm{m}^{2}, m_{0}=252.326 \mathrm{~kg} \cdot \mathrm{m}$ and Case $2, H_{s}=2.5 \mathrm{~m}$, $k=33.25 \mathrm{MN} / \mathrm{m}, \quad G_{s}=12.98 \mathrm{MN} / \mathrm{m}^{2}, \quad m_{0}=1261.63 \mathrm{~kg} \cdot \mathrm{m}$ [11]. The localized Friedlander dynamic load parameters which include the positive and the negative phase according to Eqs. (2) and (3) are: $p_{r, \max }=28.906 \mathrm{kN} / \mathrm{m}^{2}, t_{d}=0.0018 \mathrm{~s}, \alpha=0.35$, $p_{r, \min }=-7.2265 \mathrm{kN} / \mathrm{m}^{2}$ [14]. Four different locations of the localized Friedlander dynamic loading are studied in detail to determine the mid-point deflection of the rigid pavement subjected to localized Friedlander with the emphasis in the effect of the negative phase of the load.

4.1. Variation of Deflection during the Negative Phase of the Load. Figure 3 shows the variation of deflection under load as a function of time for three different values of plate thickness for Case 1. It is found that the dynamic deflection of the plate decreases as the thickness of the plate increases. Increasing the thickness of the pavement plates, from 0.18 to $0.2 \mathrm{~m}$ for a damped system $(5 \%)$ result in a decrease of the mid-point displacement by $7.9 \%$. It can be seen from Fig. 3, the maximum dynamic deflection of the pavement plate occurs just after the negative phase occurs of the Friedlander load, $t>t_{d}+t_{d-}$, for all different values of thickness considered in this work. In conclusion, the negative phase of the load contributes to increasing the maximum dynamic deflection of the system. 


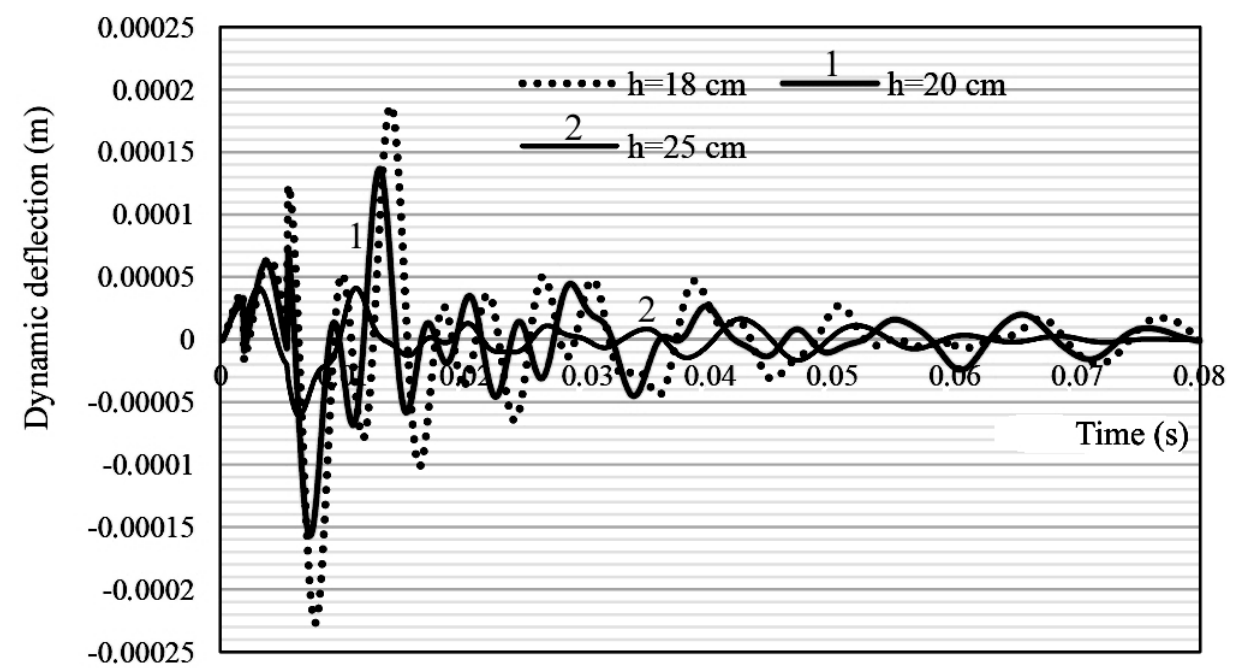

Fig. 3. Variation of the deflection directly under the localized dynamic loading versus time for different values of pavement's thickness.

4.2. Influence of the Soil Parameters on the Dynamic Response. Figure 4 shows the dynamic response of the system subjected to a Friedlander decaying dynamic loading for three types of soil conditions. At $h=0.18 \mathrm{~m}$ and $t=0.004 \mathrm{~s}$, in Case 1 , the maximum dynamic deflection of the pavement plate is $6.48092 \cdot 10^{-5} \mathrm{~m}$, while Case 2 it is $6.60447 \cdot 10^{-5} \mathrm{~m}$. It is noticed that the maximum dynamic deflection increases linearly with the increase in dynamically activated depth parameter, $H_{s}$. This result agrees very well with the previous work done by Gibigaye et al. [11].

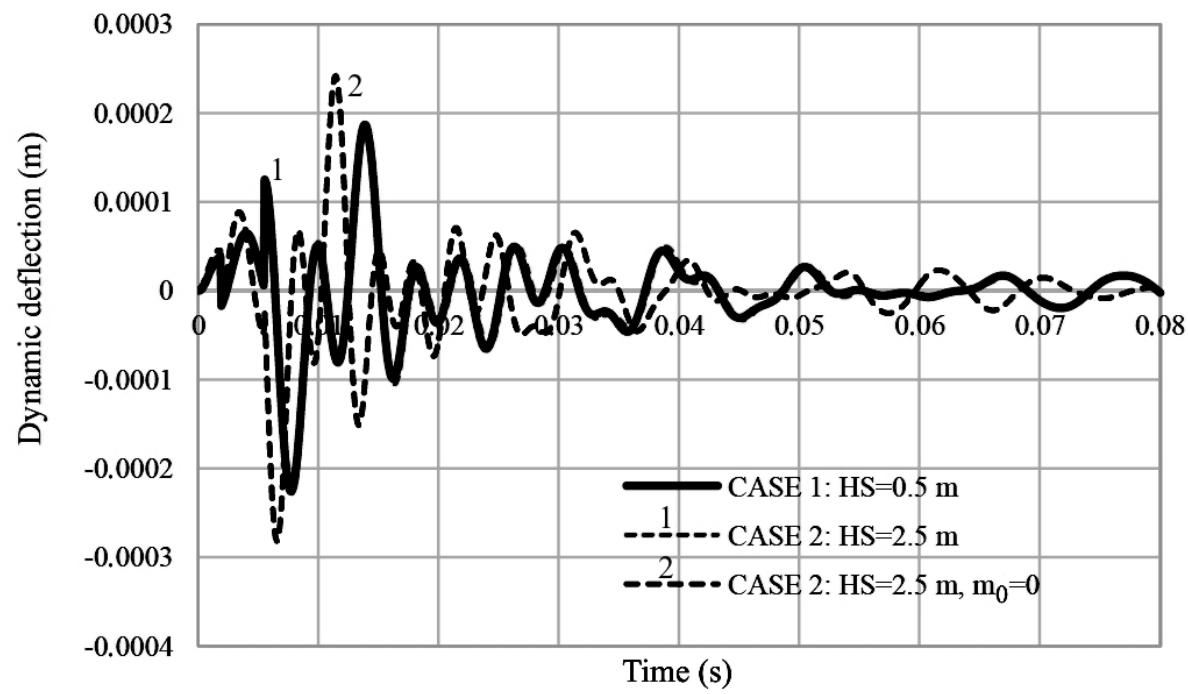

Fig. 4. Variation of the deflection directly under the localized dynamic loading versus time for different values of soil parameters.

4.3. Influence of the Inertia of the Soil Parameters on the Dynamic Response. To study the influence of the inertia of the soil factor, $m_{0}$, on the dynamic response of the pavement plate, the dynamic deflection of pavement plate during the period of 


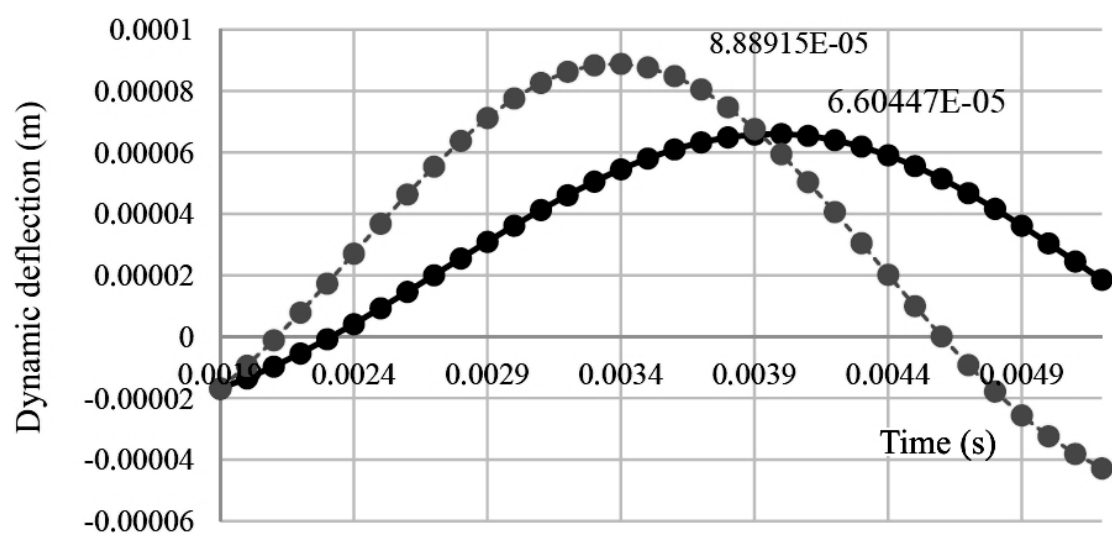

$\longrightarrow$ CASE 2: HS=2.5 m ---- PASTERNAK, $\mathrm{m}_{0}=0$

Fig. 5. The influence of the dynamic deflection of the pavement plate over the plate region due to the Friedlander decaying for two type of soil parameters conditions.

$t_{d} \leq t \leq t_{d}+t_{d}$ is computed as shown in Fig. 5. It is noticed that the mid-point deflection of the pavement plate on Pasternak foundation is $25.7 \%$ higher compared to the deflection values for the three-parameter soil. In conclusion, inertial soil dramatically reduces the dynamic response of the pavement plate when the dynamic load is at the center of the plate.

Figure 6 shows the influence of the internal bending moment during the negative phase of the load for two different type of soil conditions. It is shown that the inertial soil factor $\left(m_{0}\right)$ is an essential factor in reducing the maximum value of the internal moment in the $x$-direction for as much as $29.3 \%$.

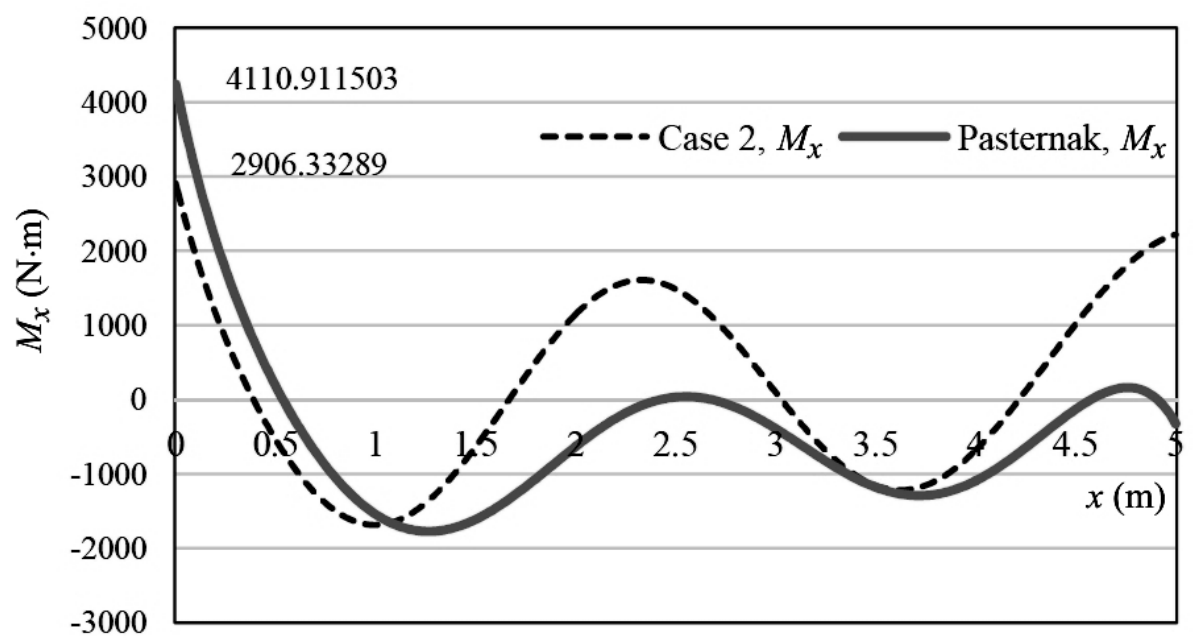

Fig. 6. Variation of the internal bending moment $\left(M_{x}\right)$ as a function of $x$ position computed during the negative phase of the load.

4.4. Effect of the Location of the Dynamic Load along the $x$-Axis. Figure 7 shows the time history of the mid-point deflection of the pavement plate subjected to localized Friedlander loading for different values of $x_{0}\left(x_{0}=0.125 a, 0.25 a, 0.375 a\right.$, and $\left.0.5 a\right)$. The results show that the dynamic deflection at mid-point increases as the position of the load closes to the middle of the pavement plate. 


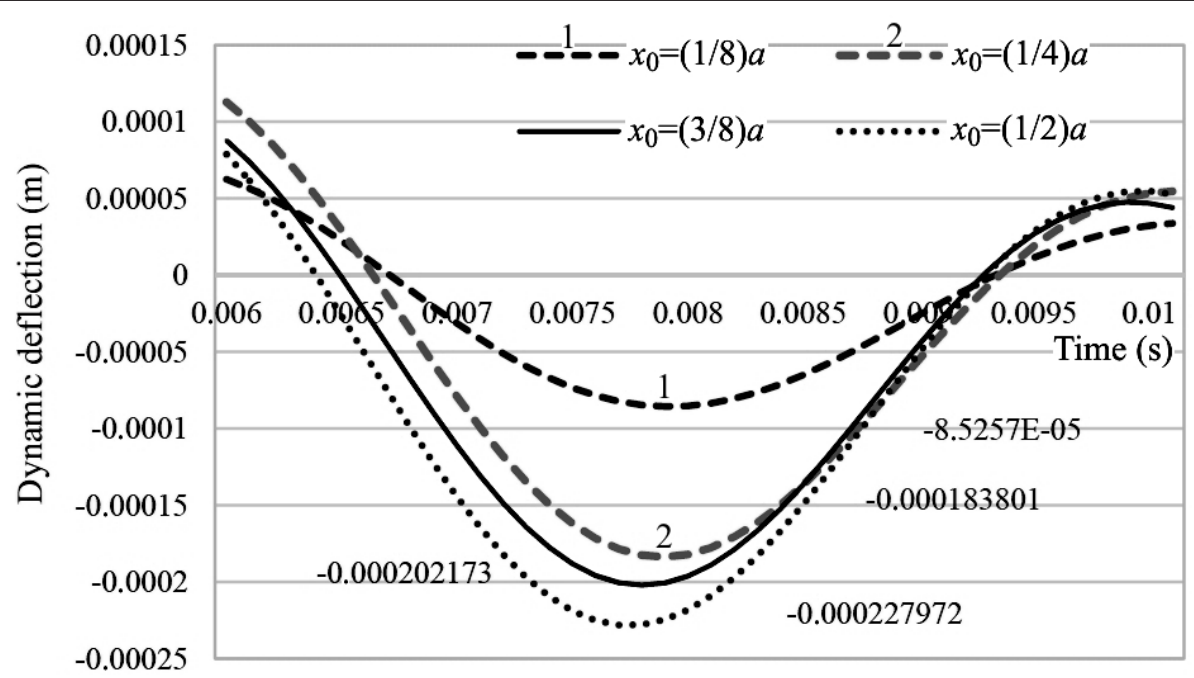

Fig. 7. Dynamic deflection time history of pavement plate subjected to localized Friedlander load for different value of $x_{0}$ (Case 2).

Conclusions. This paper dealt with some significant results from an analytical study of pavement concrete plate sitting on a three-type soil parameter foundation subjected to localized Friedlander loading which includes positive and negative phases. The pavement model consists of discrete orthotropic plates joined at the discontinuities by vertical springs representing the load transfer devices. The foundation model used in this work is the well-known Pasternak model which takes into account the interaction between soil layers, and the inertial factor of soil. The main conclusions of this study are the following:

1. The negative phase of the localized dynamic load plays an important factor in increasing the maximum dynamic deflection as well as the internal bending moment of the system.

2. The soil inertia factor $\left(m_{0}\right)$ influences the maximum dynamic deflection of the pavement plate. By including the $m_{0}$ factor into the analysis, the maximum dynamic deviation of the pavement plate was reduced by as much as $25.7 \%$.

3. The effect of the plate thickness is significant because it affects the overall behavior of the concrete plate.

4. The mid-span dynamic deflection depends on the position of the blast loading. The mid-span dynamic deflection increases as the load near the middle of the plate.

5. This study covers the pavement plates interconnected by the conditions that allow rotational and translational deformation along the boundaries. These conditions can be extended to another type of boundary conditions.

While this paper deals mainly with computational results, Yuen and Nurick [18] and Gibigaye et al. [11] reported that the dynamic response of the pavement plates is influenced significantly by the thickness of the plate and the soil parameters. That approach provides a satisfactory correlation and creates a better understanding of dynamic responses of the pavement plate on three-type of soil parameters.

Acknowledgments. The research has been supported by the Director General of Research and Development Reinforcement, Ministry of Research Technology and the Higher Education Republic of Indonesia, contract No. 035/KM/PNT/2018.

1. S. M. Kim, Dynamic Response of Pavement Systems to Moving Loads, University of Texas at Austin (1996). 
2. J. Uzan and R. L. Lytton, "Analysis of pressure distribution under falling weight deflectometer loading," J. Transp. Eng., 116, No. 2, 246-250 (1990).

3. L. Sun, "Analytical dynamic displacement response of rigid pavements to moving concentrated and line loads," Int. J. Solids Struct., 43, Nos. 14-15, 4370-4383 (2006).

4. L. Sun and B. Greenberg, "Dynamic response of linear systems to moving stochastic sources," J. Sound Vib., 229, No. 4, 957-972 (2000).

5. J. T. Kenney, "Steady-state vibrations of beam on elastic foundation for moving load," J. Appl. Mech., 21, 359-364 (1954).

6. M. R. Taheri, Dynamic Response of Plates to Moving Loads, Ph.D. Thesis, Purdue University, West Lafayette, IN (1986).

7. S. M. Kim and J. M. Roesset, Dynamic Response of Pavement Systems to Moving Loads, Research Report 1422-2, Center for Transportation Research, University of Texas at Austin (1996).

8. L. Sun, "Time-harmonic elastodynamic Green function of plates for line loads," $J$. Sound Vib., 246, No. 2, 337-348 (2001).

9. E. C. Ting, J. Genin, and J. H. Ginsberg, "A general algorithm for the moving mass problem," J. Sound Vib., 33, No. 1, 49-58 (1974).

10. G. Pan and S. N. Atluri, "Dynamic response of finite sized elastic runways subjected to moving loads: A couple BEM/FEM approach," Int. J. Numer. Meth. Eng., 38, No. 18, 3143-3166 (1995).

11. M. Gibigaye, C. P. Yabi, and I. E. Alloba, "Dynamic response of a rigid pavement plate based on an inertial soil," Int. Sch. Res. Notices, Article ID 4975345, 1-9 (2016), DOI: $10.1155 / 2016 / 4975345$.

12. S. W. Alisjahbana and W. Wangsadinata, "Dynamic analysis of rigid roadway pavement under traffic loads with variable velocity," Interact. Multiscale Mech., 5, No. 2, 105-114 (2012).

13. S. W. Alisjahbana and W. Wangsadinata, "Response of damped orthotropic stiffened plates subjected to a stepped triangular blast loading," Procedia Engineer., 14, 989-996 (2011).

14. S. Susler, H. S. Turkmen, and Z. Kazanci, "The nonlinear dynamic behaviour of tapered laminated plates subjected to blast loading," Shock Vib., 19, No. 6, 1235-1255 (2012).

15. S. E. Rigby, A. Tyas, T. Bennett, et al., "The negative phase of the blast load," Int. J. Protect. Struct., 5, No. 1, 1-19 (2014).

16. P. Pevzner, T. Weller, and A. Berkovits, "Further modification of Bolotin method in vibration analysis of rectangular plates," AIAA J., 38, No. 9, 1725-1729 (2000).

17. S. W. Alisjahbana and W. Wangsadinata, "Numerical dynamic analysis of orthotropic plates under localized blast loading," in: Proc. of the 2nd Australasia and South-East Asia Structural Engineering and Construction (Bangkok, Thailand, 2014), Isec-Press (2014), pp. 115-120.

18. S. C. K. Yuen and G. N. Nurick, "The significance of the thickness of a plate when subjected to localized blast loads," in: Proc. of the 16th Int. Symp. on Military Aspects of Blast and Shock (Oxford, UK, 2000), pp. 491-499. 